\title{
SKIN CANCER
}

\section{Benefiting from CTLA-4 blockade -a genetic rationale}

Blockade of the cytotoxic T-lymphocyteassociated antigen 4 (CTLA-4) checkpoint with ipilimumab is effective in prolonging overall survival in $20-25 \%$ of patients with advanced-stage melanoma. However, why only a subset of patients benefits from CTLA-4 blockade is not known. Now, Jedd Wolchok, Timothy Chan et al. have described the genetic basis of sensitivity to immunotherapy in melanoma.

The working hypothesis, explains Wolchok, was that "tumours with an abundance of 'passenger' mutations look less like normal cells and may be more amenable to immunotherapy." Wholeexome sequencing was performed on tumour specimens and matched blood samples. The identified mutationrelated neoantigens were analysed for their immunogenicity in vitro using bioinformatic tools and in vivo using T cells from the same patients; "a new way to interpret how somatic mutations in tumours influence the response of the immune system," states Chan.

Good responders had four-times the number of mutations than patients who did not respond to ipilimumab, and were characterized by the presence of common stretches of mutated sequences (neoepitope signature) homologous to those of pathogens. Thus, mutations might cause tumour cells to resemble pathogens, promoting a greater immune response. Wolchok and Chan agree that the next step is "to validate the neoepitope signature to inform physicians on the likelihood of success of an immunotherapy."

Alessia Errico

Original article Snyder, A. et al. Genetic basis for clinical response to CTLA-4 blockade in melanoma. N. Engl. J. Med. doi:10.1056/NEJMoa1406498 